\title{
A simple mass-action model predicts genome-wide protein timecourses from mRNA trajectories during a dynamic response in two strains of Saccharomyces cerevisiae
}

Sidney Kuo, ${ }^{1,4}$ Jarrett D. Egertson, ${ }^{2}$ Gennifer E. Merrihew, ${ }^{2}$ Michael J. MacCoss, ${ }^{2}$ Daniel A. Pollard, ${ }^{3}$ Scott A. Rifkin ${ }^{1, *}$

1. Section of Ecology, Behavior, and Evolution. Division of Biological Sciences, University of California, San Diego.

2. Department of Genome Sciences, University of Washington.

3. Biology Department, Western Washington University

4. current affiliation: Illumina, San Diego, California

*. To whom correspondence should be addressed: sarifkin@ucsd.edu

Running title: Predicting variation in dynamic protein expression

Keywords: dynamics, transcriptome, proteome, variation, yeast, pheromone

\section{Abstract}

Although mRNA is a necessary precursor to protein, several studies have argued that the relationship between mRNA and protein levels is often weak. This claim undermines the functional relevance of conclusions based on quantitative analyses of mRNA levels, which are ubiquitous in modern biology from the single gene to the whole genome scale. Furthermore, if post-translational processes vary between strains and species, then comparative studies based on mRNA alone would miss an important driver of diversity. However, gene expression is dynamic, and most studies examining relationship between mRNA and protein levels at the genome scale have analyzed single timepoints. We measure yeast gene expression after pheromone exposure and show that, for most 
genes, protein timecourses can be predicted from mRNA timecourses through a simple, gene-specific, generative model. By comparing model parameters and predictions between strains, we find that while mRNA variation often leads to protein differences, evolution also manipulates protein-specific processes to amplify or buffer transcriptional regulation.

\section{Introduction}

Nearly two decades of genome-scale analyses of gene expression variation have resulted in a broad characterization of the location and activities of genetic variants affecting mRNA abundances. Expression quantitative trait locus (eQTL) mapping experiments have revealed that mRNA abundance variation results from a large number of gene-specific genetic variants located within the locus of the varying gene, as well as a smaller number of genetic variants that act on many genes from distant locations in the genome (Yvert et al. 2003; Doss et al. 2005; Gibson and Weir 2005; Andrie et al. 2014; Pai et al. 2015; Gilad et al. 2008). Allele-specific expression measurements in F1 hybrids confirmed that variation in mRNA abundances is the result of both cis-acting (allele-specific) and trans-acting (non-allele-specific) genetic variation (Wittkopp et al. 2004). Integrating causal studies of mRNA variation into analyses of higher phenotypic variation promised to be a way to mechanistically explain how polymorphisms lead to phenotypic differences (Schadt et al. 2005).

However, while the genetics of mRNA abundance variation has advanced, its relevance to higher-level phenotypic variation is still unclear. Quantitative measurements of protein levels have shown that steady state mRNA abundances are 
not necessarily good predictors of relative protein expression across genes (Taniguchi et al. 2010; Lackner et al. 2012; Vogel and Marcotte 2012; Edfors et al. 2016; Fortelny et al. 2017), highlighting the role that gene-specific translation rates and protein stabilities can play in protein expression. This disconnect has been found not just across genes, but across individuals and strains as well. Although the reliability and sensitivity of quantitative proteomic technologies still lag behind their mRNA counterparts, initial proteomic surveys of natural variation in steady state protein levels have found that mRNA variation is not well correlated with protein variation (Minagawa et al. 2008; Khan et al. 2013; Chick et al. 2016; Consoli et al. 2002; Cenik et al. 2015; Battle et al. 2015). A mass-spectroscopy study of genetic variation in yeast underlying steady state protein levels for highly expressed genes found that a minority of variants were linked to mRNA variation (Foss et al. 2011). Other studies of the same yeast strains using fluorescent protein levels instead of mass-spectroscopy found that around half of genetic variation affecting protein levels also affects mRNA levels (Parts et al. 2014; Albert et al. 2014a), suggesting that this disconnect between mRNA and protein variation is not simply the result of technical limitations of mass-spectrometry. These studies imply that a specific class of genetic variants acts directly on protein levels to impact phenotypic variation. If variation in mRNA levels is only weakly associated with variation in protein levels, then mRNA differences would have limited relevance to downstream phenotypes (Yang et al. 2017), despite the fact that most genome-scale, comparative gene expression studies have been performed at the mRNA level (Brem et al. 2002; Rockman and Kruglyak 2006) . 
Genetic variants could affect protein expression through translation (initiation, elongation, and termination), protein folding, stability, and degradation. These effects could act in cis through variants within the locus of the gene or could act in trans through auto-regulation or the action of other genes. For translation rates, trans-acting polymorphisms have been found to be more common than cis-acting ones in yeast (Torabi and Kruglyak 2011; McManus et al. 2014). Genetic variants could also act at both the mRNA and protein levels, reinforcing or compensating for each other (Khan et al. 2013; McManus et al. 2014; Artieri and Fraser 2014; Albert et al. 2014b).

Many of the studies that document a disconnect between mRNA and protein levels across genes or across strains/individuals are based on snapshot measurements of cells (Zheng et al. 2010; Roberts et al. 2000) or organisms at steady state. As a consequence, their conclusions about the relationship between mRNA and protein may not be generalizable to common, dynamic situations such as development, disease progression, or response to environmental fluctuations. Moreover, timecourse data can reveal regulatory connections within cells that are not readily apparent even from analyses of large knockout panels (Gonçalves et al. 2017).

\section{Results}

To determine (a) whether mRNA levels are a good proxy for protein levels during the dynamic response of an organism to an environmental stimulus (Liu et al. 2016) and (b) whether differences in mRNA levels between strains translate into differences in protein levels, we profiled the transcriptomes and proteomes of two strains of Saccharomyces cerevisiae during their response to pheromone. mRNA levels in strains S288c and YJM145 have previously been shown to differ at 30 minutes after exposure to alpha 
factor pheromone (Zheng et al. 2010) and ribosome loading and transcriptional differences have been compared between these strains at steady state (Sun et al. 2016). We collected mRNA samples at $0,0.5,1,1.6,2.4,3.2,4.8,6.4$, and 8 hours after exposure and protein samples at $0,1.6,3.2,4.8,6.4$, and 8 hours for each strain in three biological replicates with mRNA and protein collected from the same flasks. We measured genome-wide levels of mRNA by RNA-seq and protein levels by quantitative mass spectrometry (Chapman et al. 2014).

Even during this response to a specific environmental signal, gene expression was pervasive genome-wide throughout the timecourse. We detected transcripts for 6,231 genes at one or more time points in at least one strain and 6,078 genes with transcripts at one or more timepoints in both strains of which a shared set of 5,595 genes could be analyzed through our DESeq2-based pipeline (Love et al. 2014). This comprises approximately $85 \%$ of yeast genes (Supplemental Methods). 2,345 different proteins were detected, and all but two were present at every timepoint in both strains (Supplemental Methods). This is approximately $35 \%$ of the genome. Proteins that are present in low abundances may not be detected via mass spectrometry (Bantscheff et al. 2007; Peshkin et al. 2015), which may partially account for fewer proteins being present than as detected by RNA-seq.

Although large numbers of genes had detectable levels of mRNA or protein, not all were affected by pheromone induction. We tested for significant induction or repression by checking whether at least one timepoint was significantly different from time 0 (FDR < 0.05) (Spies et al. 2019). With some variation between strains, around $25 \%$ of analyzed mRNA and $34 \%$ of analyzed proteins were significantly induced or 
repressed, with the majority shared between strains (Table 1; Figure 1). The classification of genes as significantly induced or repressed and/or different between strains at 1 hour post-induction were not, in general, representative of analyses over the whole timecourse (Figure 2).

Figure 1

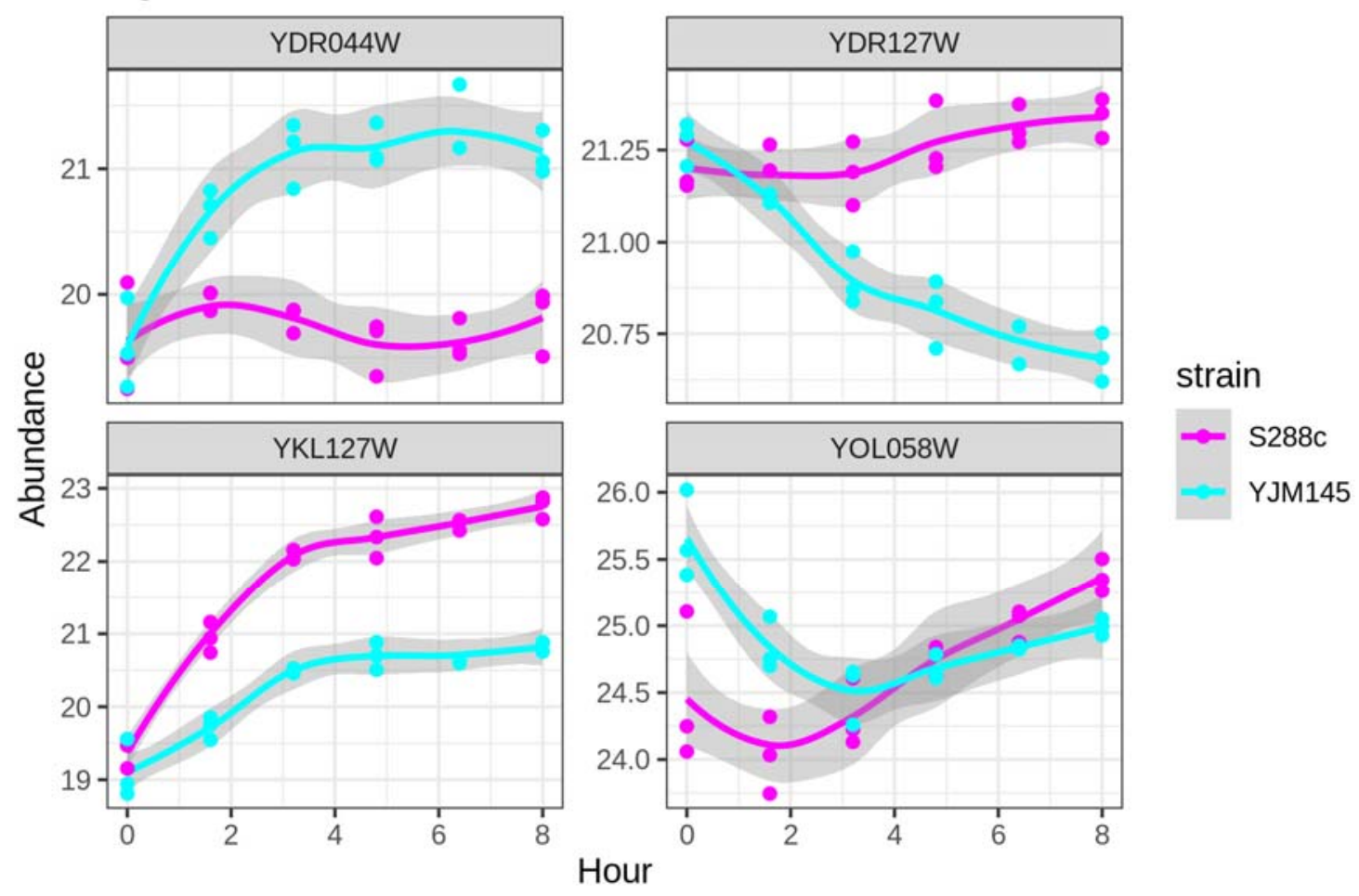

Figure 1: Example protein trajectories. Protein abundances were plotted over time, with the color denoting the strain. Dots represent actual experimentally measured time points, whereas the curves are the loess-smoothed fits of the linear model with shaded $95 \%$ confidence intervals, which represent our best estimate of average abundance at that time point. The trajectories for these four genes were identified to be significantly different between strains by a likelihood ratio test (see below). Top-left: YDR044W (HEM13) is involved in the heme biosynthetic pathway. Top-right: YDR127W (ARO1) is involved in chromisate biosynthesis, which is the precursor to aromatic amino acids. 
Bottom-left: YKL127W (PGM1) codes for phosphoglucomutase, which converts glucose-1-phosphate to glucose-6-phosphate, a process necessary for hexose metabolism. Bottom-right: YOL058W (ARG1) is an enzyme in the arginine biosynthesis pathway.

Table 1: Number of genes induced or repressed via RNA-seq and protein-MS

\begin{tabular}{|l|l|l|}
\hline & $\begin{array}{l}\text { mRNA (Total 5,595 } \\
\text { genes detected) }\end{array}$ & $\begin{array}{l}\text { Protein (Total 2345 } \\
\text { genes detected) }\end{array}$ \\
\hline S288c & $1,274(22.8 \%)$ & $793(33.8 \%)$ \\
\hline YJM145 & $1,676(30.0 \%)$ & $796(33.9 \%)$ \\
\hline Both & $900(16.1 \%)$ & $497(21.2 \%)$ \\
\hline
\end{tabular}


Figure 2
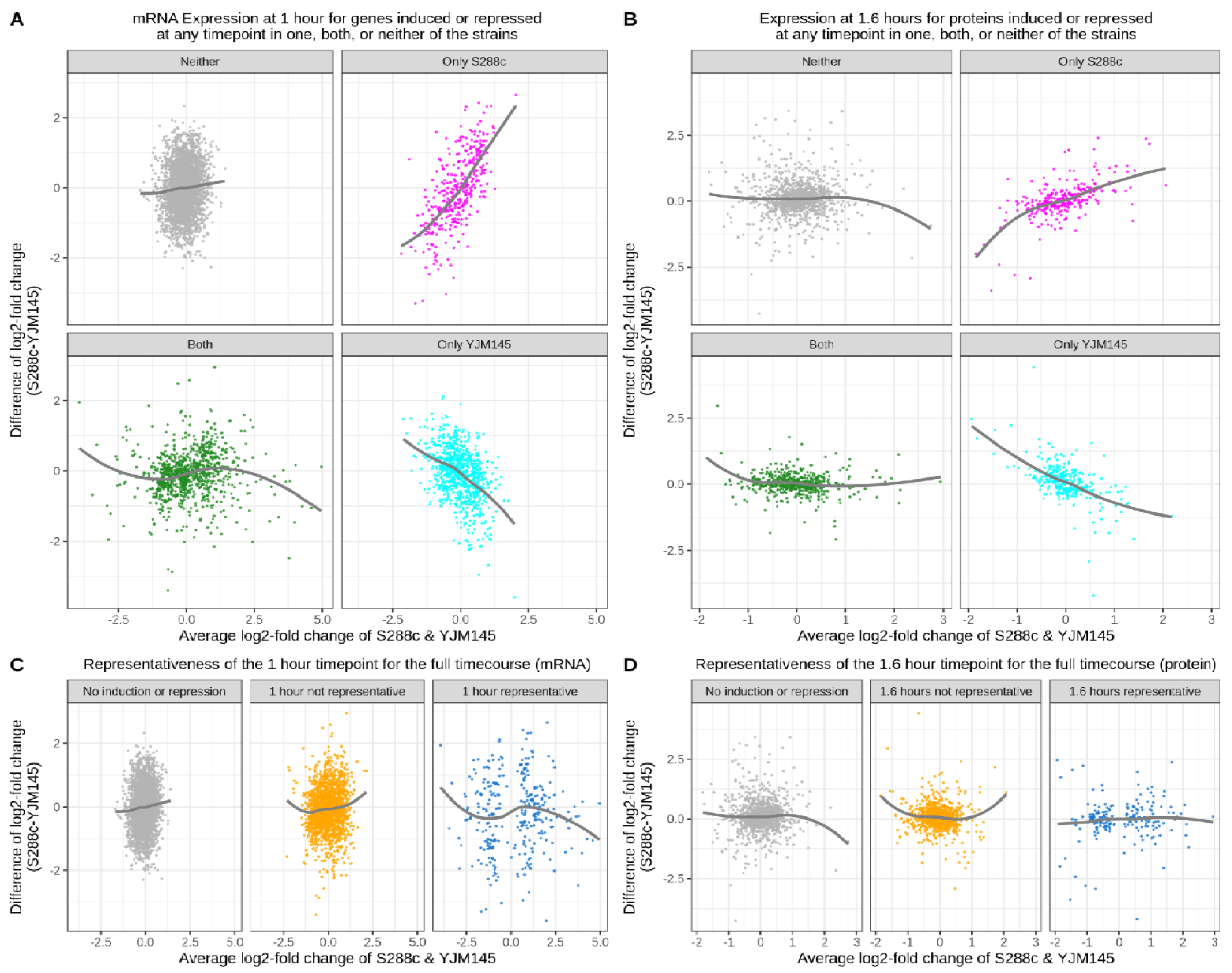

Figure 2. Studies comparing one timepoint to a baseline misclassify differential expression during a dynamic response. (A) MA plots of mRNA expression for the two strains at 1 hour after pheromone exposure separated into whether the mRNA is significantly induced or repressed during the timecourse in one, both, or neither strain. Grey lines are loess fits through the data as guides to the eye. (B) Like (A) except for protein expression at 1.6 hours after induction. (C) Like (A) except separated by whether the induction at 1 hour was representative of the whole timecourse. (D) Like (C) except for protein at 1.6 hours. 
Across genes, the correlation between strains for mRNA is 0.49 at 1 hour postinduction and for protein is 0.48 at 1.6 hours post-induction. However, despite seemingly low correlations, if we test for differences between strains over entire mRNA or protein trajectories by evaluating the significance of strain $x$ timepoint interactions in a likelihood ratio test, we detect 3.9\% (217 genes) of mRNA trajectories being significantly different between strains (FDR<0.05), and 4.6\% (108 genes) of protein trajectories being significantly different $(F D R<0.05)$. For mRNA, expression trajectories of genes involved in translation (including ribosomal genes) tended to be more divergent than expected while protein divergences were dominated by genes involve in amino-acid synthesis (Supplemental Data S1). These tend to be the categories of genes that are differentially expressed at the two levels. There were only 14 genes which were different between strains for both mRNA and protein. These numbers may be conservative since the false negative rate for mRNA and protein differences is not necessarily the same, but if we rank the $p$-values of the likelihood ratio tests for between strain differences, there is not a noticeable relationship between those for mRNA and protein (Supplemental Figure S1). This suggests that post-transcriptional regulation plays an important role, both in maintaining protein levels while mRNA differs, and in affecting protein levels without significant changes in mRNA trajectories. Large differences in mRNA do not necessarily lead to large differences in protein between strains.

If we restrict our focus to only those genes that significantly change during the response in both RNA and protein in either strain, the mean correlation between mRNA and protein trajectories is 0.19 , although significantly changing genes are enriched for 
higher correlations (Supplemental Figure S2). The low correlation is in line with the literature (Christiano et al. 2014; Maier et al. 2009) and corroborates the idea that taking only the magnitude and direction of mRNA differential expression is a poor predictor of the behavior of its corresponding protein product, presumably because it does not take into account properties like protein degradation rates. Indeed, even qualitatively, mRNA and protein are not necessarily congruent. When the mRNA changes significantly during the timecourse, only around $2 / 3$ of proteins follow the direction of this change. (Figure 3). Even among the significantly changing protein targets of STE12 - a transcriptional activator that is known to respond to pheromone - mRNA and protein change in the same direction only for 79 of 130 targets in s288c. This suggests that (a) even qualitative conclusions drawn from dynamic changes in mRNA abundance are not always representative of the behavior in protein levels and that (b) generalizations about protein behavior based on mRNA measurements at single timepoints can be strikingly mistaken.

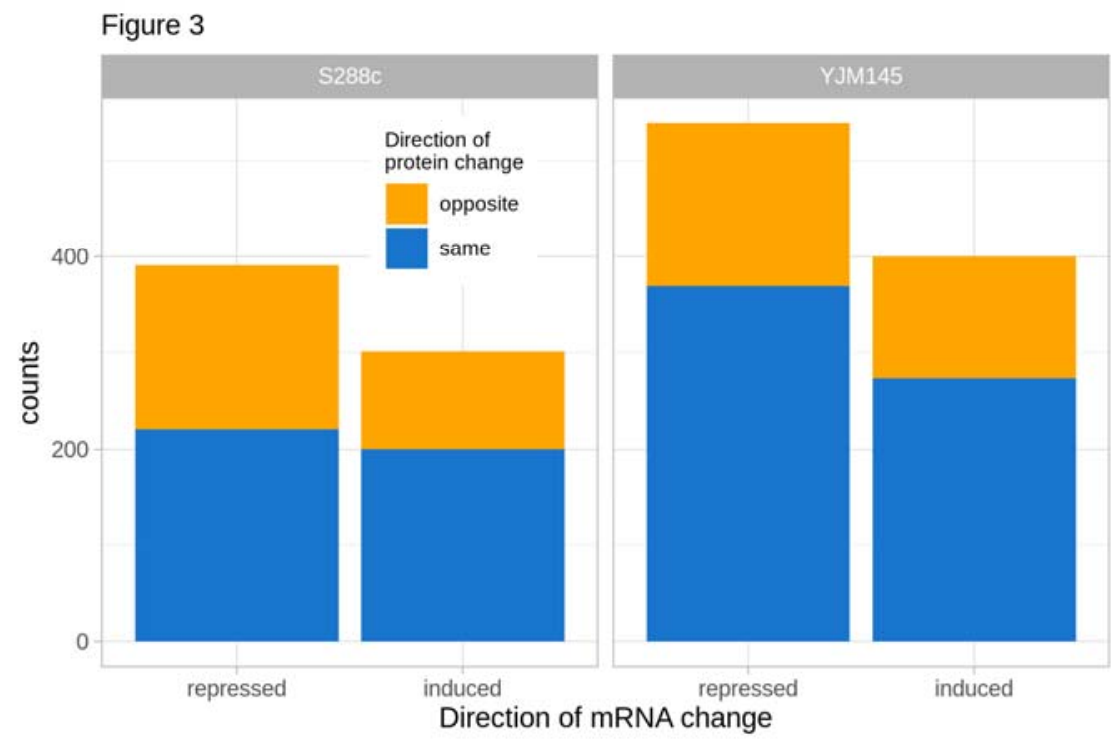

Figure 3. Concordance of induction or repression between mRNA and protein. 
In order to bridge the disconnect between mRNA and protein levels and to study variation in the protein production process, we fit a mass action model to our protein data (Lee et al. 2011) of the form $d p / d t=k_{s} R(t-\tau)-k_{D} P(t)$, where $k_{s}$ is the protein synthesis rate after pheromone exposure, $R(t-\tau)$ is the measured amount of mRNA at some amount of time $\tau$ before time $t, k_{D}$ is the protein degradation rate after pheromone exposure, and $P(t)$ is the amount of protein at time $t$. Because individual parameter estimates are often poorly constrained in systems biology models (Gutenkunst et al. 2007; Erguler and Stumpf 2011), we estimated their joint posterior distribution for each strain separately and for a model where both strains shared the same synthesis and degradation values (Supplemental Methods). This Bayesian framework allowed us to compare these rates between strains along with the structure of their covariation while accounting for the uncertainties in the parameter estimates (Gutenkunst et al. 2007; McElreath 2018). 
bioRxiv preprint doi: https://doi.org/10.1101/805846; this version posted October 27, 2019. The copyright holder for this preprint (which was not certified by peer review) is the author/funder, who has granted bioRxiv a license to display the preprint in perpetuity. It is made available under aCC-BY-NC-ND 4.0 International license.

\section{Figure 4}

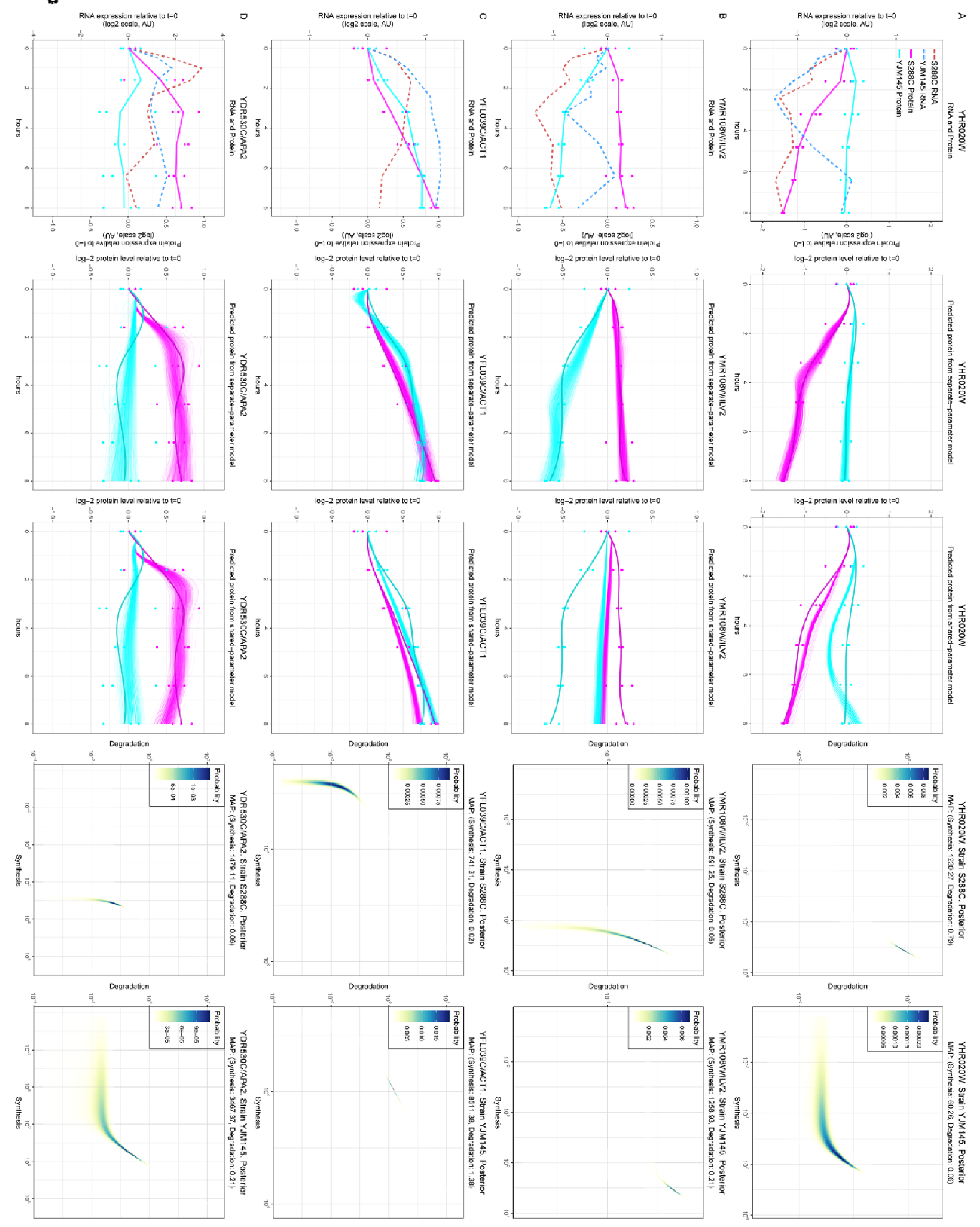

Figure 4: RNA, protein, and parameter variation can take several different forms. The figure is described rotated into landscape orientation. From left to right, the 5 panels in 
each row are: (1) the mean mRNA and protein trajectories with protein data plotted as filled circles. Dotted lines are RNA (red: S288C; blue: YJM145) and solid lines are protein (magenta: S288C; cyan: YJM145). The values are on a log scale relative to the value at $\mathrm{t}=0$. (2) 500 trajectories simulated from the posterior of the separate-parameter model. Spline fits to the actual protein data are shaded in a slightly darker color than the simulations. Protein data is plotted as filled circles. As in (1), the values are on a log scale relative to the value at $t=0$. (3) Same as (2) except simulated trajectories are generated from the shared-parameter model. (4) Heat map of the posterior density estimate for S288C. (5) Heat map of the posterior density estimate for YJM145. (A): YHR020W has significantly different mRNA, protein, and parameters; (B) YMR108W (ILV2) has significantly different protein and parameters but not mRNA trajectories; (C) YFL039C (ACT1) has significantly different mRNA trajectories and parameters but not protein trajectories; (D) YDR530C (APA2) has shared parameter values between strains but significantly different mRNA and protein trajectories.

The model fit the data well. For each gene and strain we simulated trajectories from the posterior (Figure 4). The mean normalized root mean squared error was less than $2 \%$ for approximately $60 \%$ of the genes and less than $10 \%$ for $98 \%$ of them. At the maximum a posteriori estimates, protein synthesis rates during the pheromone response range are log-normally distributed with the middle $50 \%$ ranging from 1.9 to 22.5 per minute. The middle $50 \%$ of protein half lives range from 1.9 to 10.3 hours with $13.1 \%$ (S288c) and 10.7\% (YJM145) of proteins with half-lives less than one hour. The predicted protein half-lives were also approximately log-normally distributed (Belle et al. 
2006), although we found an overabundance of short lived proteins and an underrepresentation of long-lived ones (Christiano et al. 2014) (Figure 5; Supplemental Figure S3). While this could be the result of MS bias, which tends to only pick up more abundant proteins, pheromone response induces substantial physiological changes such as cell cycle arrest, which may lead to active protein degradation for certain proteins compared with steady-state growth conditions (Bardwell 2005).

Figure 5

A

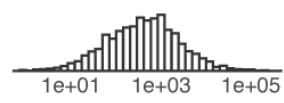

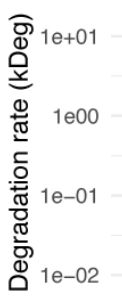

$\begin{array}{ccc}1 e+01 & 1 e+03 & 1 e+05 \\ \text { Synthesis rate (kSyn) }\end{array}$
B

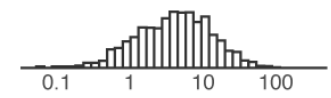

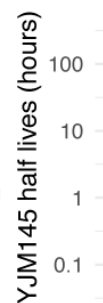

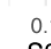

S288c half lives (hours)

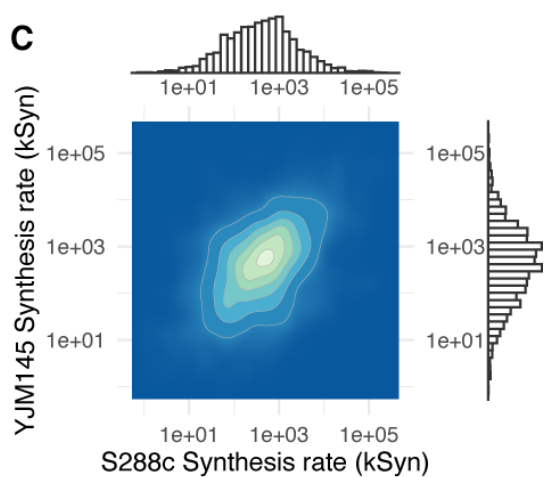

Figure 5: Maximum a posteriori parameter estimates. (A) Density plot and histograms of synthesis rate and degradation rate pairs for both strains together. (B) Density plot and histograms of protein half-lives. (C) Density plot and histograms of estimated protein synthesis rates during pheromone response.

In order to lead to differences in protein trajectories, mutations must alter either RNA trajectories or biochemical parameters such as the translation and degradation rates we modeled here. We found that strain-specific protein synthesis and degradation rates gave demonstrably better protein trajectory predictions than a shared parameter model based on WAIC (Watanabe 2010; McElreath 2018) for 300 of the 2,316 genes that had good data for both RNA and protein (FDR $<0.05$; Figure 5). Genes involved in 
small molecule metabolic processes were most likely to have divergent parameters between strains (FDR < 1E-13; Supplemental Data S1), particularly those active in the mitochondria (Eden et al. 2009). This apparent divergence in the parameters controlling mitochondrial protein dynamics is consistent with other results showing that YJM145 is divergent from S288c-like lab strains in mitochondrial expression (Sun et al. 2016).

Of these 2,316 genes, 406 significantly diverged between strains (FDR<0.05) in either RNA, protein, or parameters or a combination of factors (Figures 4,5). Over half of these only had detectable differences in parameter values. For 71 genes, dominated by genes involved in translation, the divergence was in RNA alone, only 1 gene showed divergence in RNA and protein trajectories without a concomitant change in parameters, and 60 genes, including those involved in biosynthesis, had significant divergence in protein trajectories and parameters. These tests have different statistical power, but we should be better able to detect RNA differences than protein differences and so we likely overestimate the number of genes diverging in RNA alone and underestimate those diverging in protein or protein and parameters. In either case, these numbers make it clear both that studies focusing on the mRNA level alone will miss a large fraction of potentially functional divergence and that parameters of these dynamic biological processes can vary between strains without leading to substantial differences in protein expression. These phenotypically neutral parameter shifts in our study place cells from the two strains in different regions of the parameter space and, we hypothesize, mean that the strains have different potentials to respond to new physiological or evolutionary pressures.

\section{Discussion}


After an initial explosion of microarray and then RNA-seq studies exploring genome-wide variation in mRNA levels (Rifkin et al. 2003; Landry et al. 2007; Gilad et al. 2005; Ranz et al. 2003; Meiklejohn et al. 2003; Brem et al. 2002), an increasing number of studies are uncovering genetic variation affecting post-transcriptional processes (Schaefke et al. 2018; Pai et al. 2015; Pai and Gilad 2014; Battle et al. 2015). These include RNA splicing (Bradley et al. 2012; Barbosa-Morais et al. 2012; Manning and Cooper 2017), poly-adenylation (Xiao et al. 2016), RNA decay (which also affects RNA trajectories) (Andrie et al. 2014), and protein-specific processes like those investigated here (McManus et al. 2014; Wu et al. 2013; Foss et al. 2007; Horvatovich et al. 2014; Stark et al. 2014; Sun et al. 2016; Albert et al. 2014b). This variation complicates the link between DNA variation and phenotypic variation, and it is not yet clear whether there are general, systematic patterns that distinguish the kinds of biological processes that are likely to be affected by transcriptional variation versus post-transcriptional variation.

Our results show that, for any given gene, mRNA levels are not necessarily reliable predictors of protein levels even during an active response to a stimulus and that differences in mRNA abundances between strains do not necessarily translate into differences at the protein level. Much of the documented mRNA expression variation between strains and species is probably screened off from selection by processes acting downstream. This allows transcriptional variation to build up and form a reservoir that can fuel future evolutionary change .

However, although the relationship between mRNA and protein levels at a given timepoint can be tenuous, protein trajectories can be predicted from mRNA by a simple, 
generative, mass-action model that uses physiologically plausible parameter values to represent the post-transcriptional processes of protein synthesis and degradation.

These parameter values can differ between strains, and they certainly depend upon cellular context (Bardwell 2005). Modeling the data in this way highlights the fact that protein production itself is a buffering process that can smooth over transcriptional bursts (Raj et al. 2006) and temper differences in mRNA trajectories. When protein degradation rates are slow compared to those of mRNA, then protein production will act as if it is integrating mRNA levels over time, and tweaks to protein synthesis and degradation parameters could keep two strains' protein levels similar to each other despite mRNA divergence. If, as often postulated for enzyme kinetics, the cellular effect of a protein is a saturating function of protein level (Kacser and Burns 1981; Savageau 1976), then this could allow synthesis and degradation rates to drift and build up evolutionary potential as long as they jointly maintain at least a minimum protein level. On the flip side, reconfiguring cellular state to face a new environmental challenge could require gene-specific shifts in the balance of synthesis and degradation so that the cell's complement of proteins can be rapidly turned over without having to wait for the effects of changes in mRNA numbers to percolate to the protein level. We find cases where the mRNA levels do not appreciably change after pheromone exposure while the protein increases or declines.

Our estimated posterior densities (Figure 5) show that, in general, coordinated changes to protein synthesis and degradation rates can maintain consistent protein trajectory predictions from an mRNA timecourse. However, most of the genes for which we found protein differences did not differ in their mRNA trajectories, and so buffering is 
not necessarily the main task of parameter variation. Instead, modifications to protein synthesis and degradation rates can directly generate differences at the protein level from the same mRNA substrate. These three knobs - mRNA levels, synthesis rates, and degradation - can be changed dynamically and either in a coordinated manner or independently of each other and give evolution the ability to finely tune protein trajectories to meet specific cellular exigencies. Over evolutionary time, they can drift, loosely bounded by stabilizing selection, and build up a reservoir of untested variation in a population that could fuel future evolutionary change. 


\section{Methods}

\section{Strains and experimental conditions}

The two S. cerevisiae strains used in this study were S288c and YJM145. Both are mating type a with the following genes knocked out via the loxP system (Gueldener et al. 2002): URA3, HO, AMN1, and BAR1. In both strains, FIG1 had been tagged with yECitrine using the Ca.URA3 marker for other studies. Strains were grown in $50 \mathrm{~mL}$ YPD overnight as a starter culture, with the experimental culture being in $1 \mathrm{~L}$ YPD in a $3 \mathrm{~L}$ Fernbach flask to provide proper aeration. Experimental cultures were diluted to an OD of $\sim 0.1$ and incubated for 2 hours before inducing with pheromone. Pheromone was added to achieve a final concentration of $50 \mathrm{nM}$ in the media. Samples taken for the mRNA were at hours $0,0.5,1,1.6,2.4,3.2,4.8,6.4,8$ where hour 0 is taken immediately before induction. Samples for the protein MS were taken from hours $0,1.6$, 3.2, 4.8, 6.4, 8. Fifty $\mathrm{mL}$ of each sample was taken from the flask at each time point, spun down, and flash frozen in liquid nitrogen. Experiments were performed on three separate days with the RNA and protein handled together afterwards.

Total RNA extraction of all the samples was done using the Ambion RiboPure kit. Multiplexed RNA-Seq libraries were generated from the 3' end of transcripts according to a published protocol (Kadoki et al. 2017) The protocol produces mRNA reads proportional to the number of transcripts independent of transcript length. Sequencing was done on an Illumina HiSeq4000 using single reads 50 base pairs in length. Quantitative MS was performed by the MacCoss laboratory including processing of raw MS data (see Supplemental Methods). 


\section{Processing and statistical methods}

Processing of RNA-Seq data was done on Galaxy, using BWA to align reads to the $S$. cerevisiae reference genome (R64-2-1, 2015-01-13) and htseq-count to extract read counts for each transcript(Li and Durbin 2009; Anders et al. 2015; Afgan et al. 2016). Estimation of gene counts was performed using DESeq2 (Love et al. 2014) with a generalized linear model parameterized as count $\sim$ timepoint + strain + timepoint:strain, with timepoint being a factor variable(Love et al. 2014). Likelihood ratio tests between the strains use the form count $\sim$ timepoint + strain as the reduced model, which would not include differences in the pre-induction time point between the strains.

Pre-processing of MS intensity values was done using Skyline, followed by sample normalization and log transformation via MSStats (Choi et al. 2014; Egertson et al. 2015) (Supplemental Methods). A general linear model using log intensities for each protein that follows the same form as the RNA-seq model was fit to the data, and the likelihood ratio test was performed the same way.

All analysis and modeling was performed in $R$ ( $R$ Core Team 2019). Although each of our RNA replicates corresponded to a protein replicate from the same flask, including this pairing in the modeling did not improve the fit in general. We estimated posterior densities for each gene for a model where the synthesis and degradation parameters were shared between strains and one where they were allowed to differ using a weakly-informative prior on the parameters (Supplemental Methods). All fits were on a log scale as errors are thought to be symmetrical for protein levels on a log scale.

\section{Data access}


All raw RNA-seq data generated in this study have been submitted to the NCBI Gene Expression Omnibus (GEO; https://www.ncbi.nlm.nih.gov/geo/) under accession number GSE131211. All mass spectrometry data generated in this study have been submitted to Panorama public at the URL https://panoramaweb.org/KuoRifkin.url and has the ProteomeXchange ID PXD015745. The complete processed data, R code, and outputs from the analyses will be available from the UCSD library digital collection archive at https://library.ucsd.edu/dc/

\section{Acknowledgements}

This project was supported by National Science Foundation grant NSF MCB-1517482

to SAR and NSF MCB-1518314 to DP. SK was partially supported on NIH training grant T32GM007240-39A1.

\section{Author Contributions}

SK, SAR, and DP designed the study. SK performed the experiments. JE, GM, MM performed the MS measurements. SK and SAR analyzed the data and wrote the paper.

\section{Disclosure Declaration}

The authors report no conflicts of interest. 
bioRxiv preprint doi: https://doi.org/10.1101/805846; this version posted October 27, 2019. The copyright holder for this preprint (which was not certified by peer review) is the author/funder, who has granted bioRxiv a license to display the preprint in perpetuity. It is made available under aCC-BY-NC-ND 4.0 International license.

\section{Supplemental material}

Supplemental Methods

Supplemental Figures (S1-S3)

Supplemental Data S1. Zip archive with the results of the GOrilla (Eden et al. 2009) GO analyses for between-strain differences of mRNA, protein, and parameters for Process, Function, and Component ontologies. 


\section{References}

Afgan E, Baker D, van den Beek M, Blankenberg D, Bouvier D, Čech M, Chilton J, Clements D, Coraor N, Eberhard C, et al. 2016. The Galaxy platform for accessible, reproducible and collaborative biomedical analyses: 2016 update. Nucleic Acids Research 44: W3-W10.

Albert FW, Muzzey D, Weissman JS, Kruglyak L. 2014a. Genetic Influences on Translation in Yeast ed. R.B. Brem. PLoS Genetics 10: e1004692.

Albert FW, Treusch S, Shockley AH, Bloom JS, Kruglyak L. 2014b. Genetics of singlecell protein abundance variation in large yeast populations. Nature 506: 494-497.

Anders S, Pyl PT, Huber W. 2015. HTSeq--a Python framework to work with highthroughput sequencing data. Bioinformatics (Oxford, England) 31: 166-9.

Andrie JM, Wakefield J, Akey JM. 2014. Heritable variation of mRNA decay rates in yeast. Genome Res 24: 2000-2010.

Artieri CG, Fraser HB. 2014. Evolution at two levels of gene expression in yeast. Genome Res 24: 411-421.

Bantscheff M, Schirle M, Sweetman G, Rick J, Kuster B. 2007. Quantitative mass spectrometry in proteomics: a critical review. Analytical and Bioanalytical Chemistry 389: 1017-1031.

Barbosa-Morais NL, Irimia M, Pan Q, Xiong HY, Gueroussov S, Lee LJ, Slobodeniuc V, Kutter C, Watt S, Çolak R, et al. 2012. The Evolutionary Landscape of Alternative Splicing in Vertebrate Species. Science 338: 1587-1593.

Bardwell L. 2005. A walk-through of the yeast mating pheromone response pathway. Peptides 26: 339-350.

Battle A, Khan Z, Wang SH, Mitrano A, Ford MJ, Pritchard JK, Gilad Y. 2015. Impact of regulatory variation from RNA to protein. Science 347: 664-667.

Belle A, Tanay A, Bitincka L, Shamir R, O'Shea EK. 2006. Quantification of protein halflives in the budding yeast proteome. Proceedings of the National Academy of Sciences of the United States of America 103: 13004-9.

Bradley RK, Merkin J, Lambert NJ, Burge CB. 2012. Alternative Splicing of RNA Triplets Is Often Regulated and Accelerates Proteome Evolution. PLOS Biology 10: e1001229.

Brem RB, Yvert G, Clinton R, Kruglyak L. 2002. Genetic Dissection of Transcriptional Regulation in Budding Yeast. Science 296: 752-755. 
Cenik C, Cenik ES, Byeon GW, Grubert F, Candille SI, Spacek D, Alsallakh B, Tilgner H, Araya $\mathrm{CL}$, Tang $\mathrm{H}$, et al. 2015. Integrative analysis of RNA, translation, and protein levels reveals distinct regulatory variation across humans. Genome Res 25: $1610-1621$.

Chapman JD, Goodlett DR, Masselon CD. 2014. Multiplexed and data-independent tandem mass spectrometry for global proteome profiling. Mass Spectrometry Reviews 33: 452-470.

Chick JM, Munger SC, Simecek P, Huttlin EL, Choi K, Gatti DM, Raghupathy N, Svenson KL, Churchill GA, Gygi SP. 2016. Defining the consequences of genetic variation on a proteome-wide scale. Nature 534: 500-505.

Choi M, Chang C-Y, Clough T, Broudy D, Killeen T, MacLean B, Vitek O. 2014. MSstats: an $R$ package for statistical analysis of quantitative mass spectrometrybased proteomic experiments. Bioinformatics 30: 2524-2526.

Christiano R, Nagaraj N, Fröhlich F, Walther TC. 2014. Global Proteome Turnover Analyses of the Yeasts S. cerevisiae and S. pombe. Cell Reports 9: 1959-1965.

Consoli L, Lefèvre A, Zivy M, de Vienne D, Damerval C. 2002. QTL analysis of proteome and transcriptome variations for dissecting the genetic architecture of complex traits in maize. Plant Mol Biol 48: 575-581.

Doss S, Schadt EE, Drake TA, Lusis AJ. 2005. Cis-acting expression quantitative trait loci in mice. Genome Res 15: 681-691.

Eden E, Navon R, Steinfeld I, Lipson D, Yakhini Z. 2009. GOrilla: a tool for discovery and visualization of enriched $\mathrm{GO}$ terms in ranked gene lists. BMC Bioinformatics 10: 48 .

Edfors F, Danielsson F, Hallström BM, Käll L, Lundberg E, Pontén F, Forsström B, Uhlén M. 2016. Gene•specific correlation of RNA and protein levels in human cells and tissues. Molecular Systems Biology 12: 883.

Egertson JD, MacLean B, Johnson R, Xuan Y, MacCoss MJ. 2015. Multiplexed peptide analysis using data-independent acquisition and Skyline. Nature Protocols 10: 887-903.

Erguler K, Stumpf MPH. 2011. Practical limits for reverse engineering of dynamical systems: a statistical analysis of sensitivity and parameter inferability in systems biology models. Mol BioSyst 7: 1593-1602.

Fortelny N, Overall CM, Pavlidis P, Freue GVC. 2017. Can we predict protein from mRNA levels? Nature 547: E19-E20. 
Foss EJ, Radulovic D, Shaffer SA, Goodlett DR, Kruglyak L, Bedalov A. 2011. Genetic Variation Shapes Protein Networks Mainly through Non-transcriptional Mechanisms. PLOS Biology 9: e1001144.

Foss EJ, Radulovic D, Shaffer SA, Ruderfer DM, Bedalov A, Goodlett DR, Kruglyak L. 2007. Genetic basis of proteome variation in yeast. Nat Genet 39: 1369-1375.

Gibson G, Weir B. 2005. The quantitative genetics of transcription. Trends in Genetics 21: $616-623$.

Gilad Y, Rifkin SA, Bertone P, Gerstein M, White KP. 2005. Multi-species microarrays reveal the effect of sequence divergence on gene expression profiles. Genome Res 15: 674-680.

Gilad Y, Rifkin SA, Pritchard JK. 2008. Revealing the architecture of gene regulation: the promise of eQTL studies. Trends in genetics 24: 408-415.

Gonçalves E, Nakic ZR, Zampieri M, Wagih O, Ochoa D, Sauer U, Beltrao P, SaezRodriguez J. 2017. Systematic Analysis of Transcriptional and Posttranscriptional Regulation of Metabolism in Yeast. PLOS Computational Biology 13: e1005297.

Gueldener U, Heinisch J, Koehler GJ, Voss D, Hegemann JH. 2002. A second set of loxP marker cassettes for Cre-mediated multiple gene knockouts in budding yeast. Nucleic Acids Research 30: 23e-223.

Gutenkunst RN, Waterfall JJ, Casey FP, Brown KS, Myers CR, Sethna JP. 2007. Universally Sloppy Parameter Sensitivities in Systems Biology Models. PLoS Comput Biol 3: e189.

Horvatovich P, Franke L, Bischoff R. 2014. Proteomic Studies Related to Genetic Determinants of Variability in Protein Concentrations. J Proteome Res 13: 5-14.

Kacser H, Burns JA. 1981. The molecular basis of dominance. Genetics 97: 639-666.

Kadoki M, Patil A, Thaiss CC, Brooks DJ, Pandey S, Deep D, Alvarez D, von Andrian UH, Wagers AJ, Nakai K, et al. 2017. Organism-Level Analysis of Vaccination Reveals Networks of Protection across Tissues. Cell 171: 398-413.e21.

Khan Z, Ford MJ, Cusanovich DA, Mitrano A, Pritchard JK, Gilad Y. 2013. Primate Transcript and Protein Expression Levels Evolve Under Compensatory Selection Pressures. Science 342: 1100-1104.

Lackner DH, Schmidt MW, Wu S, Wolf DA, Bähler J. 2012. Regulation of transcriptome, translation, and proteome in response to environmental stress in fission yeast. Genome Biology 13: R25. 
Landry CR, Lemos B, Rifkin SA, Dickinson WJ, Hartl DL. 2007. Genetic Properties Influencing the Evolvability of Gene Expression. Science 317: 118-121.

Lee MV, Topper SE, Hubler SL, Hose J, Wenger CD, Coon JJ, Gasch AP. 2011. A dynamic model of proteome changes reveals new roles for transcript alteration in yeast. Molecular Systems Biology 7.

http://www.nature.com/msb/journal/v7/n1/full/msb201148.html (Accessed January 8, 2013).

Li H, Durbin R. 2009. Fast and accurate short read alignment with Burrows-Wheeler transform. Bioinformatics 25: 1754-1760.

Liu Y, Beyer A, Aebersold R. 2016. On the Dependency of Cellular Protein Levels on mRNA Abundance. Cell 165: 535-550.

Love MI, Huber W, Anders S. 2014. Moderated estimation of fold change and dispersion for RNA-seq data with DESeq2. Genome Biology 15: 550.

Maier T, Güell M, Serrano L. 2009. Correlation of mRNA and protein in complex biological samples. FEBS Letters 583: 3966-3973.

Manning KS, Cooper TA. 2017. The roles of RNA processing in translating genotype to phenotype. Nature Reviews Molecular Cell Biology 18: 102-114.

McElreath R. 2018. Statistical rethinking: A Bayesian course with examples in $R$ and Stan. Chapman and Hall/CRC.

McManus CJ, May GE, Spealman P, Shteyman A. 2014. Ribosome profiling reveals post-transcriptional buffering of divergent gene expression in yeast. Genome Res 24: $422-430$.

Meiklejohn CD, Parsch J, Ranz JM, Hartl DL. 2003. Rapid evolution of male-biased gene expression in Drosophila. PNAS 100: 9894-9899.

Minagawa H, Yamashita T, Honda M, Tabuse Y, Kamijo K, Tsugita A, Kaneko S. 2008. Comparative Analysis of Proteome and Transcriptome in Human Hepatocellular Carcinoma using 2D-DIGE and SAGE. Protein J 27: 409-419.

Pai AA, Gilad Y. 2014. Comparative studies of gene regulatory mechanisms. Current Opinion in Genetics \& Development 29: 68-74.

Pai AA, Pritchard JK, Gilad Y. 2015. The Genetic and Mechanistic Basis for Variation in Gene Regulation. PLOS Genetics 11: e1004857.

Parts L, Liu Y-C, Tekkedil MM, Steinmetz LM, Caudy AA, Fraser AG, Boone C, Andrews BJ, Rosebrock AP. 2014. Heritability and genetic basis of protein level variation in an outbred population. Genome Res 24: 1363-1370. 
Peshkin L, Wühr M, Pearl E, Haas W, Freeman RM, Gerhart JC, Klein AM, Horb M, Gygi SP, Kirschner MW. 2015. On the Relationship of Protein and mRNA Dynamics in Vertebrate Embryonic Development. Developmental Cell 35: 383394.

R Core Team. 2019. R: A Language and Environment for Statistical Computing. R Foundation for Statistical Computing, Vienna, Austria https://www.R-project.org/.

Raj A, Peskin CS, Tranchina D, Vargas DY, Tyagi S. 2006. Stochastic mRNA Synthesis in Mammalian Cells. PLoS Biol 4: e309.

Ranz JM, Castillo-Davis CI, Meiklejohn CD, Hartl DL. 2003. Sex-Dependent Gene Expression and Evolution of the Drosophila Transcriptome. Science 300: 17421745.

Rifkin SA, Kim J, White KP. 2003. Evolution of gene expression in the Drosophila melanogaster subgroup. Nat Genet 33: 138-144.

Roberts CJ, Nelson B, Marton MJ, Stoughton R, Meyer MR, Bennett HA, He YD, Dai H, Walker WL, Hughes TR, et al. 2000. Signaling and Circuitry of Multiple MAPK Pathways Revealed by a Matrix of Global Gene Expression Profiles. Science 287.

Rockman M V., Kruglyak L. 2006. Genetics of global gene expression. Nature Reviews Genetics 7: 862-872.

Savageau MA. 1976. Biochemical systems analysis: a study of function and design in molecular biology. Addison-Wesley Pub. Co., Advanced Book Program.

Schadt EE, Lamb J, Yang X, Zhu J, Edwards S, GuhaThakurta D, Sieberts SK, Monks S, Reitman M, Zhang C, et al. 2005. An integrative genomics approach to infer causal associations between gene expression and disease. Nat Genet 37: 710717.

Schaefke B, Sun W, Li Y-S, Fang L, Chen W. 2018. The evolution of posttranscriptional regulation. Wiley Interdisciplinary Reviews: RNA 9: e1485.

Spies D, Renz PF, Beyer TA, Ciaudo C. 2019. Comparative analysis of differential gene expression tools for RNA sequencing time course data. Brief Bioinform. https://academic.oup.com/bib/advance-article/doi/10.1093/bib/bbx115/4364840 (Accessed January 17, 2019).

Stark AL, Jr RJH, Gorsic LK, Antao NN, Wong SS, Chung SH, Gill DF, Im HK, Myers JL, White KP, et al. 2014. Protein Quantitative Trait Loci Identify Novel Candidates Modulating Cellular Response to Chemotherapy. PLOS Genetics 10: e1004192.

Sun X, Wang Z, Guo X, Li H, Gu Z. 2016. Coordinated Evolution of Transcriptional and Post-Transcriptional Regulation for Mitochondrial Functions in Yeast Strains. PLOS ONE 11: e0153523. 
Taniguchi Y, Choi PJ, Li G-W, Chen H, Babu M, Hearn J, Emili A, Xie XS. 2010. Quantifying E. coli proteome and transcriptome with single-molecule sensitivity in single cells. Science 329: 533-538.

Torabi N, Kruglyak L. 2011. Variants in SUP45 and TRM10 Underlie Natural Variation in Translation Termination Efficiency in Saccharomyces cerevisiae. PLOS Genetics 7: e1002211.

Vogel C, Marcotte EM. 2012. Insights into the regulation of protein abundance from proteomic and transcriptomic analyses. Nature Reviews Genetics 13: 227-232.

Watanabe S. 2010. Asymptotic Equivalence of Bayes Cross Validation and Widely Applicable Information Criterion in Singular Learning Theory. Journal of Machine Learning Research 11: 3571-3594.

Wittkopp PJ, Haerum BK, Clark AG. 2004. Evolutionary changes in cis and trans gene regulation. Nature 430: 85-88.

Wu L, Candille SI, Choi Y, Xie D, Jiang L, Li-Pook-Than J, Tang H, Snyder M. 2013. Variation and genetic control of protein abundance in humans. Nature 499: 7982.

Xiao M-S, Zhang B, Li Y-S, Gao Q, Sun W, Chen W. 2016. Global analysis of regulatory divergence in the evolution of mouse alternative polyadenylation. Molecular Systems Biology 12: 890.

Yang J-R, Maclean CJ, Park C, Zhao H, Zhang J. 2017. Intra and Interspecific Variations of Gene Expression Levels in Yeast Are Largely Neutral: (Nei Lecture, SMBE 2016, Gold Coast). Mol Biol Evol 34: 2125-2139.

Yvert G, Brem R, Whittle J, Akey J, Foss E. 2003. Trans-acting regulatory variation in Saccharomyces cerevisiae and the role of transcription factors. Nature Genetics. http://www.nature.com/ng/journal/v35/n1/abs/ng1222.html.

Zheng W, Zhao H, Mancera E, Steinmetz LM, Snyder M. 2010. Genetic analysis of variation in transcription factor binding in yeast. Nature 464: 1187-1191. 